\title{
Sulphur abundances in disk stars: A correlation with silicon ${ }^{\star}$
}

\author{
Y. Q. Chen ${ }^{1,2}$, P. E. Nissen ${ }^{2}$, G. Zhao ${ }^{1}$, and M. Asplund ${ }^{3,4}$ \\ 1 National Astronomical Observatories, Chinese Academy of Sciences, Beijing 100012, PR China \\ 2 Institute of Physics and Astronomy, University of Aarhus, 8000 Aarhus C, Denmark \\ 3 Uppsala Astronomical Observatory, Box 515, 751 20, Sweden \\ ${ }^{4}$ Present address: Research School of Astronomy and Astrophysics, Australian National University, \\ Mount Stromlo Observatory, Cotter Road, Weston, ACT 2611, Australia
}

Received 10 January 2002 / Accepted 15 May 2002

\begin{abstract}
We have performed new determinations of sulphur and silicon abundances for a sample of 26 disk stars based on high-resolution, high signal-to-noise spectra. The results indicate a solar $[\mathrm{S} / \mathrm{Fe}]$ for $[\mathrm{Fe} / \mathrm{H}]>-0.3$, below which $[\mathrm{S} / \mathrm{Fe}]$ increases to $\sim 0.25 \mathrm{dex}$ at $[\mathrm{Fe} / \mathrm{H}]=-1.0$. We find that there is a good correlation between $[\mathrm{S} / \mathrm{H}]$ and $[\mathrm{Si} / \mathrm{H}]$, indicating the same nucleosynthetic origin of the two elements. It seems that the ratio of sulphur to silicon does not depend on metallicity for $[\mathrm{Fe} / \mathrm{H}]>-1.0$. The implications of these results on models for the nucleosynthesis of $\alpha$-capture elements and the chemical evolution of the Galaxy are discussed.
\end{abstract}

Key words. stars: abundances - stars: late-type - galaxy: evolution

\section{Introduction}

The trend of $[\mathrm{O} / \mathrm{Fe}]$ vs. $[\mathrm{Fe} / \mathrm{H}]$ has recently been paid much attention because works by different authors yield very dissimilar results. Specifically, Israelian et al. $(1998,2001)$ and Boesgaard (1999) derived oxygen abundances from OH UV lines and the infrared $\mathrm{O}$ I triplet and found a linear rise of $[\mathrm{O} / \mathrm{Fe}]$ to $1.0 \mathrm{dex}$ when the metallicity decreases to $[\mathrm{Fe} / \mathrm{H}]=-3.0 \mathrm{dex}$. Some doubts have, however, recently been cast on the correctness of these results from calculations based on the new generation of 3D hydrodynamical model atmospheres (Asplund \& García Pérez 2001). Other studies (e.g. Nissen et al. 1992; Nissen et al. 2001) based on the forbidden line at $\lambda 630.03 \mathrm{~nm}$ suggest, on the other hand, a more or less flat trend of $[\mathrm{O} / \mathrm{Fe}]$ in the metallicity range of $-2.0<[\mathrm{Fe} / \mathrm{H}]<-1.0$. Concerning other well-studied $\alpha$-capture elements, e.g. $\mathrm{Mg}, \mathrm{Si}$ and $\mathrm{Ca}$, earlier observed trends of $[\alpha / \mathrm{Fe}]$ versus $[\mathrm{Fe} / \mathrm{H}]$ (e.g. Ryan et al. 1996) seemed to be in good agreement with a plateau of $[\alpha / \mathrm{Fe}] \sim 0.4$ dex. In particular, Fuhrmann (1998) found a clearly flat $[\mathrm{Mg} / \mathrm{Fe}]$ ratio for $[\mathrm{Fe} / \mathrm{H}]<-0.8$ based on a LTE abundance analysis of $\mathrm{Mg}$ and $\mathrm{Fe}$. However, the recent work of Stephens \& Boesgaard (2002) based on Keck spectra of more than 50 halo and disk stars suggests a quasi-linear trend of increasing $[\alpha / \mathrm{Fe}]$ with decreasing $[\mathrm{Fe} / \mathrm{H}]$ (see their Fig. 20), but we note that their average $[\alpha / \mathrm{Fe}]$ is only about $\sim 0.4 \mathrm{dex}$ at $[\mathrm{Fe} / \mathrm{H}]=-3.0$. In addition, Idiart \& Thévenin (2000) have

\footnotetext{
Send offprint requests to: P. E. Nissen, e-mail: pen@i fa.au. dk

* Based on observations carried out at National Astronomical Observatories (Xinglong, PR China).
}

found a complicated structure in $[\mathrm{Mg} / \mathrm{Fe}]$ and $[\mathrm{Ca} / \mathrm{Fe}]$ vs. $[\mathrm{Fe} / \mathrm{H}]$ based on a non-LTE re-analysis of equivalent widths from various sources. Although the scatter is significant for $[\mathrm{Fe} / \mathrm{H}]<-1.0$, their results are consistent with plateau-like mean trends at $\sim+0.2-+0.3$ for both $[\mathrm{Mg} / \mathrm{Fe}]$ and $[\mathrm{Ca} / \mathrm{Fe}]$ for halo stars. Since different trends of $[\alpha / \mathrm{Fe}]$ vs. $[\mathrm{Fe} / \mathrm{H}]$ correspond to different nucleosynthesis histories of the $\alpha$ elements, a clarification of the divergency on the trend of $[\mathrm{O} / \mathrm{Fe}]$ vs. $[\mathrm{Fe} / \mathrm{H}]$ is very important for many astrophysical fields including stellar evolution theory, nucleosynthesis theory, the formation and chemical evolution of the Galaxy, and the age of the Galaxy and the Universe.

Another $\alpha$ element, sulphur, is highly interesting as an independent tracer of the nucleosynthesis of $\alpha$ elements and a well-established trend of $[\mathrm{S} / \mathrm{Fe}]$ vs. $[\mathrm{Fe} / \mathrm{H}]$ could be helpful in clarifing the discrepancy of the $[\mathrm{O} / \mathrm{Fe}]$ ratio. Furthermore, sulphur is of particular interest in connection with studies of the chemical enrichment of damped Ly $\alpha$ systems being one of the few elements that is not depleted onto dust (Centurión et al. 2000). Unfortunately, sulphur has been ignored for many years probably due to the lack of measurable lines in the optical spectra. Presently, our knowledge of sulphur abundances is limited to only a few studies: Clegg et al. (1981), François (1987, 1988) and recent works by Israelian \& Rebolo (2001) and Takada-Hidai et al. (2002). Again, the disagreement on the $[\mathrm{S} / \mathrm{Fe}]$ ratio at low metallicities appears: François (1987, 1988 ) suggested the $[\mathrm{S} / \mathrm{Fe}]$ ratio to be constant for stars with $-1.6<[\mathrm{Fe} / \mathrm{H}]<-1.0$, while Israelian \& Rebolo (2001) favor a linear rise of $[\mathrm{S} / \mathrm{Fe}]$ ratio with decreasing $[\mathrm{Fe} / \mathrm{H}]$ in the 
metallicity range of $-3.0<[\mathrm{Fe} / \mathrm{H}]<-0.6$. With a smaller slope ( -0.25 vs. -0.46 in Israelian \& Rebolo paper) the work of Takada-Hidai et al. (2002) support a linear trend of [S/Fe].

Sulphur and iron abundances in disk stars are usually better determined than in the case of halo stars. The sulphur lines have a suitable strength for accurate abundance determination and Fe II lines, which have very small non-LTE effects, can be used for deriving the iron abundance. Therefore, the $[\mathrm{S} / \mathrm{Fe}]$ vs. $[\mathrm{Fe} / \mathrm{H}]$ trend can be more reliably established. With this advantage, the determination of sulphur abundances in disk stars with $[\mathrm{Fe} / \mathrm{H}]>-1.0$ is important especially from the nucleosynthesis theory point of view. The comparison of the sulphur behaviour with those of other $\alpha$ elements in disk stars provides a key clue to the genuine origin of sulphur and further to the chemical evolution of the Galaxy. As the nearest even- $Z$ neighbour of sulphur in the periodic table of elements, silicon is very suitable as a representative of the $\alpha$ elements for such a comparison.

In the present work, we investigate the behaviour of sulphur in disk stars and the nucleosynthesis origin of this element. The investigation takes benefit of more accurate observations of sulphur lines, better determinations of stellar parameters, improved atmospheric models, and hence more reliable derivations of sulphur, silicon and iron abundances than in previous works (Clegg et al. 1981; François 1987, 1988), where the scatter of $[\mathrm{S} / \mathrm{Fe}]$ for disk stars is so large that it is not clear if sulphur really behaves as an $\alpha$-capture elements or not. In a following paper (Nissen et al. 2002), the study will be extented to halo stars such that the trend of $[\mathrm{S} / \mathrm{Fe}]$ will be covered for the whole metallicity range of $-3.0<[\mathrm{Fe} / \mathrm{H}]<+0.2$.

\section{Observations}

The stars were selected from the $u v b y \beta$ photometric catalogues of Olsen $(1983,1993)$ according to the criteria of $5600 \leq T_{\text {eff }} \leq$ $6600 \mathrm{~K}, 3.8 \leq \log g \leq 4.5$ and $-1.0 \leq[\mathrm{Fe} / \mathrm{H}] \leq+0.5$ with approximately equal numbers of stars in every metallicity interval of 0.1 dex. This selection aimed at obtaining a sample of stars with similar parameters in order to minimize the analysis uncertainties including the ways of determining stellar parameters and atmospheric modeling.

Spectra of stars were obtained during three observing runs: Oct. 17-22, 2000, Jan. 8-10, 2001 and Aug. 11, 2001 with the Coudé Echelle Spectrograph attached to the $2.16 \mathrm{~m}$ telescope at National Astronomical Observatories of Chinese Academy of Sciences (Xinglong, China). The detector was a Tek CCD $\left(1024 \times 1024\right.$ pixels with $24 \times 24 \mu \mathrm{m}^{2}$ each in size $)$. The red arm of the spectrograph with a 31.6 grooves $/ \mathrm{mm}$ grating was used in combination with a prism as cross-disperser, providing a good separation between the echelle orders. With a $0.5 \mathrm{~mm}$ slit (1.1 arcsec), the resolving power was on the order of 37000 . The exposure time was chosen in order to obtain a signal-tonoise around $150-250$ and the spectral coverage is $580-880 \mathrm{~nm}$ except for 3 stars with the wavelength range $550-830 \mathrm{~nm}$. As examples, three sections of the spectrum for HD 168151 and HD 22879 are shown in Figs. 1 and 2 with Fe II, SiI and S I lines marked by vertical lines. Note that HD 22879 is the most metal-poor star in this sample, and the weak S I 869.39 line was not used in deriving the sulphur abundance because

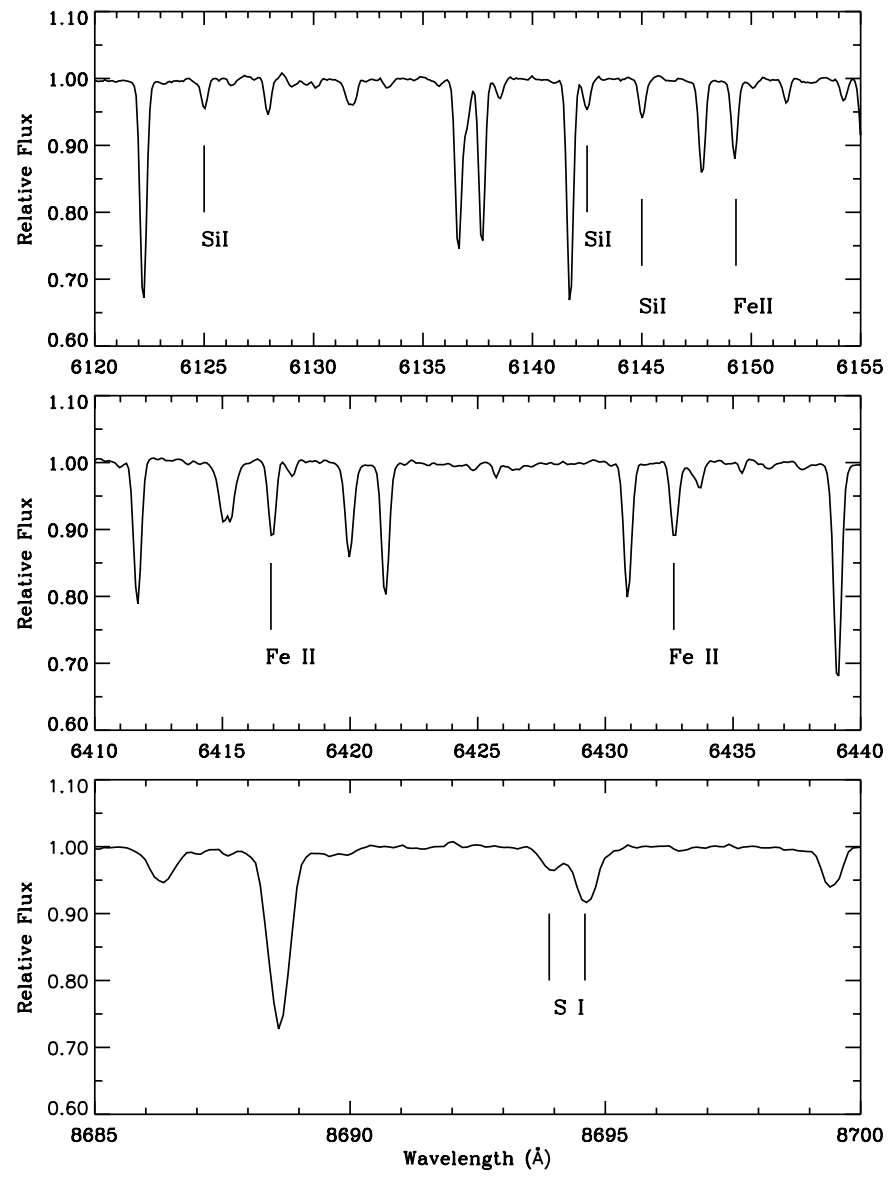

Fig. 1. Three sections of the spectrum for HD $168151\left(T_{\text {eff }}=6530 \mathrm{~K}\right.$, $\log g=4.12,[\mathrm{Fe} / \mathrm{H}]=-0.28$ ) as observed with the $2.16 \mathrm{~m}$ telescope at Xinglong Station.

the noise level in the continuum at this region is comparable with the line strength.

The data reduction, using MIDAS software, involved standard routines for order definition, background subtraction, wavelength calibration, flat-field division, spectrum extraction followed by radial velocity shift correction and continuum normalization. The equivalent widths (EWs) were measured by direct integration or Gaussian fitting depending on which method gave the more reliable value. The two S I lines at $\lambda 869.4 \mathrm{~nm}$ were separately measured using a combination of two Gaussians.

Spectroscopic binaries and a few stars with a high-rotation of $V_{\text {rot }} \sin i \geq 25 \mathrm{~km} \mathrm{~s}^{-1}$ were excluded from the sample leaving finally 26 stars for abundance analysis. Table 1 presents the star sample as well as the stellar parameters, kinematics, the equivalent widths of the $\mathrm{S}$ I lines and abundances.

\section{Analysis}

\subsection{Stellar parameters}

The effective temperature was estimated from Strömgren photometric indices (Olsen 1983, 1993) by using the $b-y$ calibration of Alonso et al. (1996). We have calculated the $E(b-y)$ for every program star using the calibrations of Olsen (1988) 

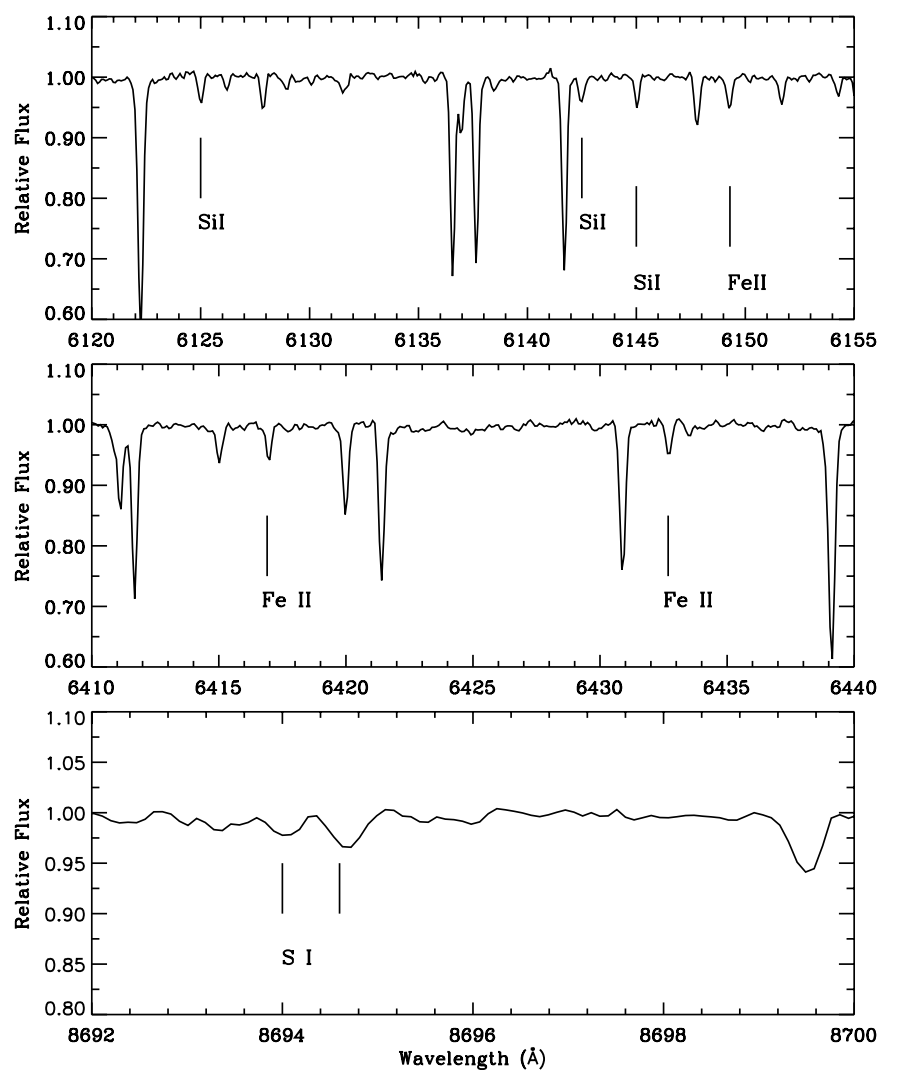

Fig. 2. Three sections of the spectrum for HD $22879\left(T_{\text {eff }}=5788 \mathrm{~K}\right.$, $\log g=4.29,[\mathrm{Fe} / \mathrm{H}]=-0.91)$ as observed with the $2.16 \mathrm{~m}$ telescope at Xinglong Station.

but found no significant reddening correction. All stars have $E(b-y)$ less than 0.010 .

$K$ magnitudes for 4 stars are available in the $2 M A S S$ survey (Finlator et al. 2000). We derived the temperature by using the $V-K$ calibrations of Alonso et al. (1996). The results are $5698 \mathrm{~K}$ for HD 22879, $6077 \mathrm{~K}$ for HD 28620, $6232 \mathrm{~K}$ for HD 77967 and $5882 \mathrm{~K}$ for HD 216106. These values are slightly lower than those derived from the $b-y$ indices (see Table 1) with a mean deviation $\langle T(V-K)-T(b-y)\rangle=$ $-66 \pm 27 \mathrm{~K}$, but they are consistent within the estimated error of the temperature determination, i.e. $\sim 100 \mathrm{~K}$. For consistency, we adopted the value from the $b-y$ indices for all stars. Furthermore, we checked that there is no significant trend of the derived iron abundance with excitation potential of low energy level of lines, supporting the adopted temperatures to within $\pm 100 \mathrm{~K}$.

As the gravity determines the degree of ionization for each element and it has a significant effect on Fe II and S I lines, a careful derivation of this parameter is important. With the available Hipparcos parallaxes, we can determine accurate gravities from the relations $g \propto \mathcal{M} / R^{2}$ and $L \propto R^{2} T_{\text {eff }}{ }^{4}$. The stars have accurate parallaxes with a relative error of $<0.05$ except for 3 stars with $\frac{\Delta \pi}{\pi} \sim 0.08$. Using the derived temperature and absolute magnitude, the mass was estimated from the stellar position in the $M_{V}-\log T_{\text {eff }}$ diagram by interpolating in the evolutionary tracks of VandenBerg et al. (2000) and the bolometric correction was interpolated from the grids of Alonso et al. (1995).

The metallicity, required in the input for temperature determination and abundance calculation, was first derived from the Strömgren $m_{1}$ index using the calibrations of Schuster \& Nissen (1989). But the final metallicity from the abundance analysis of the $\mathrm{Fe}$ II lines was used to iterate the whole procedure to full consistency. The microturbulence, $\xi_{\mathrm{t}}$, was obtained by requiring a zero slope of $[\mathrm{Fe} / \mathrm{H}]$ vs. EW based on $\mathrm{Fe}$ II lines.

The uncertainties of the stellar parameters are estimated to be around $100 \mathrm{~K}$ in $T_{\text {eff }}, 0.1 \mathrm{dex}$ in $\log g, 0.1 \mathrm{dex}$ in $[\mathrm{Fe} / \mathrm{H}]$ and $0.3 \mathrm{~km} \mathrm{~s}^{-1}$ in $\xi_{\mathrm{t}}$.

\subsection{Abundance calculations}

The abundance analysis is based on a grid of flux constant, homogeneous, LTE model atmospheres, which were computed with a recent version of the MARCS code using updated continuous opacities including UV line blanketing of millions of absorption lines and many molecular lines (Asplund et al. 1997). These models are based on the assumption of $[\alpha / \mathrm{Fe}]=$ 0.0 . Differences are only $0.05 \mathrm{dex}$ in $[\mathrm{Fe} / \mathrm{H}],-0.04 \mathrm{dex}$ in $[\mathrm{Si} / \mathrm{Fe}]$ and -0.02 in $[\mathrm{S} / \mathrm{Fe}]$ by using a model calculated with $[\alpha / \mathrm{Fe}]=0.4$ for HD 22879 , which is the most metal-poor star in our sample. Given that enhancements of $[\mathrm{S} / \mathrm{Fe}]$ and $[\mathrm{Si} / \mathrm{Fe}]$ for our sample of stars are less than $0.3 \mathrm{dex}$, the enhancement of $[\alpha / \mathrm{Fe}]$ or not in the model atmosphere has a negligible effect on the result. The abundance analysis program, EQWIDTH, was used to calculate the theoretical equivalent widths from the models, and elemental abundances were derived by requiring that the calculated equivalent widths should match the observed ones. When more than one line of the same species were measured for a star, the mean value is adopted by giving equal weight to each line.

Log $g f$ values for the S I, Si I and Fe II lines are presented in Table 2 together with solar equivalent widths measured from the Moon spectrum observed at Xinglong except for three lines with $\lambda>860 \mathrm{~nm}$. The equivalent widths for these three lines were measured from the solar flux spectrum of Kurucz et al. (1984) because the Moon spectrum observed at Xinglong does not cover the wavelength range of $\lambda>820 \mathrm{~nm}$. Solar abundances are calculated based on a solar model atmosphere computed with the same code as used for the stars. The $g f$ values from Biémont et al. (1991) (including the normalization of -0.05 dex to get agreement with lifetime measurements) are adopted for most Fe II lines. With these values, we derived a so$\operatorname{lar} \mathrm{Fe}$ abundance close to the meteorite value $\log \varepsilon_{\odot}(\mathrm{Fe})=7.50$ (Grevesse \& Sauval 1998). We obtained differential $\log g f$ values for the remaining three $\mathrm{Fe}$ II lines by forcing them to give a consistent solar abundance. The $\log g f$ values for four of the five S I lines are taken from Kurucz (2002). As shown in Table 2, the line-to-line scatter of the derived sulphur abundance is small for these four lines. From the $\lambda 604.60 \mathrm{~nm}$ line, we get a higher solar sulphur abundance. In the solar flux spectrum, this line seems to be blended with a weak nearby line at the blue edge, but we are not sure if this can account for the divergent abundance because the $\lambda 604.60 \mathrm{~nm}$ line seems to 
Table 1. Stellar basic parameters, kinematics, equivalent widths of the $\mathrm{S}$ I lines and abundances.

\begin{tabular}{|c|c|c|c|c|c|c|c|c|c|c|c|c|c|c|}
\hline \multirow[t]{2}{*}{ HD } & $T_{\mathrm{eff}}$ & $\log g$ & $\xi_{\mathrm{t}}$ & $U_{\mathrm{LSR}}$ & $V_{\mathrm{LSR}}$ & $W_{\text {LSR }}$ & \multirow{2}{*}{$\begin{array}{r}E W(1) \\
\mathrm{m} \AA \\
\end{array}$} & \multirow{2}{*}{$\begin{array}{r}E W(2) \\
m \AA ̊ \\
\end{array}$} & \multirow{2}{*}{$\begin{array}{r}E W(3) \\
\mathrm{mA} \\
\end{array}$} & \multirow{2}{*}{$\begin{array}{r}E W(4) \\
\mathrm{m} \AA \\
\end{array}$} & \multirow{2}{*}{$\begin{array}{r}E W(5) \\
\mathrm{m} \AA \\
\end{array}$} & \multirow[t]{2}{*}[\frac{\mathrm{Fe}}{\mathrm{H}}]{} & \multirow[t]{2}{*}[\frac{\mathrm{S}}{\mathrm{Fe}}]{} & \multirow[t]{2}{*}[\frac{\mathrm{Si}}{\mathrm{Fe}}]{} \\
\hline & $\mathrm{K}$ & & & & $\mathrm{km} \mathrm{s}^{-1}$ & & & & & & & & & \\
\hline 693 & 6173 & 4.11 & 1.6 & -29 & -8 & -12 & 9.8 & - & - & 10.7 & 30.1 & -0.30 & -0.06 & -0.04 \\
\hline 1461 & 5660 & 4.33 & 0.6 & 22 & -34 & 5 & - & 16.9 & - & 13.4 & 35.9 & 0.47 & -0.20 & -0.21 \\
\hline 9826 & 6119 & 4.12 & 1.9 & -38 & -17 & -8 & - & - & - & 27.8 & 56.2 & 0.12 & 0.04 & -0.03 \\
\hline 10453 & 6368 & 3.96 & 2.1 & -46 & -59 & -3 & 12.3 & 11.1 & 19.2 & 16.2 & 52.7 & -0.46 & 0.17 & 0.15 \\
\hline 13540 & 6301 & 4.12 & 1.7 & -2 & 29 & 5 & 13.1 & 20.6 & - & - & - & -0.43 & 0.19 & 0.08 \\
\hline 16895 & 6228 & 4.27 & 1.6 & 21 & 7 & 7 & 19.4 & 16.2 & - & - & 51.0 & 0.01 & 0.01 & -0.04 \\
\hline 17948 & 6455 & 4.20 & 1.6 & 21 & 14 & 20 & 13.8 & 11.9 & 22.6 & - & 43.9 & -0.26 & 0.03 & 0.02 \\
\hline 22879 & 5788 & 4.29 & 1.4 & 100 & -81 & -36 & - & - & - & - & 10.5 & -0.91 & 0.23 & 0.23 \\
\hline 28620 & 6114 & 4.11 & 1.6 & 11 & 5 & 8 & 9.9 & 9.3 & 13.2 & 9.6 & 33.4 & -0.38 & 0.06 & -0.01 \\
\hline 30652 & 6424 & 4.28 & 1.8 & 15 & -10 & 12 & 23.8 & 17.6 & - & - & - & 0.04 & -0.02 & -0.04 \\
\hline 33564 & 6276 & 4.16 & 1.9 & -30 & 11 & 3 & 24.9 & 20.7 & - & 27.5 & - & 0.05 & 0.05 & 0.04 \\
\hline 39315 & 6202 & 3.83 & 1.8 & -36 & 33 & 6 & - & 10.7 & 22.9 & 17.4 & 45.1 & -0.38 & 0.16 & 0.10 \\
\hline 49933 & 6592 & 4.21 & 1.9 & -34 & -9 & -3 & - & 12.0 & 18.3 & - & 40.5 & -0.42 & 0.10 & 0.05 \\
\hline 58461 & 6562 & 4.11 & 2.0 & 8 & 9 & -22 & - & 14.6 & - & - & 49.8 & -0.15 & -0.05 & -0.08 \\
\hline 77967 & 6329 & 4.15 & 2.0 & -74 & 4 & 11 & 12.1 & 8.5 & 15.3 & - & 35.4 & -0.46 & 0.11 & 0.11 \\
\hline 82328 & 6308 & 3.84 & 1.7 & 47 & -30 & -17 & 16.4 & 13.3 & - & 18.8 & 45.5 & -0.13 & -0.09 & -0.04 \\
\hline 84737 & 5813 & 4.12 & 1.5 & -22 & 0 & 24 & - & - & - & 14.2 & 38.2 & 0.13 & -0.10 & 0.00 \\
\hline 94388 & 6379 & 3.96 & 1.8 & -39 & -12 & -10 & 27.7 & 25.1 & - & - & 71.7 & 0.07 & 0.05 & 0.03 \\
\hline 150177 & 6061 & 3.93 & 1.7 & -4 & -19 & -18 & - & 7.4 & 14.4 & - & - & -0.63 & 0.17 & 0.10 \\
\hline 159307 & 6237 & 3.93 & 1.7 & 3 & -18 & 10 & 10.4 & 8.1 & 14.3 & - & - & -0.65 & 0.24 & 0.20 \\
\hline 162003 & 6498 & 4.02 & 2.1 & -40 & 1 & 1 & 22.6 & - & - & - & 61.2 & 0.02 & -0.08 & -0.04 \\
\hline 168151 & 6530 & 4.12 & 1.9 & -4 & -9 & -44 & - & - & 26.7 & 21.3 & 44.5 & -0.28 & 0.09 & 0.05 \\
\hline 187013 & 6298 & 4.15 & 1.9 & -47 & -2 & -17 & 18.4 & - & 26.3 & 21.6 & 41.4 & -0.02 & -0.09 & -0.04 \\
\hline 215648 & 6158 & 3.96 & 1.6 & -14 & -27 & -21 & 13.1 & 11.2 & 23.1 & - & 42.8 & -0.24 & 0.05 & 0.04 \\
\hline 216106 & 5923 & 3.74 & 1.5 & -47 & -34 & -25 & - & - & 21.6 & - & 38.2 & -0.16 & 0.04 & 0.03 \\
\hline 222368 & 6178 & 4.08 & 1.7 & -2 & -21 & -20 & 18.2 & 14.7 & - & - & 44.9 & -0.13 & 0.06 & 0.03 \\
\hline
\end{tabular}

$E W$ : (1) $\lambda 604.603 \mathrm{~nm}$; (2) $\lambda 605.267 \mathrm{~nm}$; (3) $\lambda 675.717 \mathrm{~nm}$; (4) $\lambda 869.396 \mathrm{~nm}$; (5) $\lambda 869.464 \mathrm{~nm}$.

give a consistent abundance with that derived from the other $\mathrm{S}$ I lines for most of our sample stars. The $\log g f$ value presented in Kurucz (2002) (-0.78) will give an even higher abundance than that presented in Table 2. Therefore, we derived a differential $\log g f$ value for this line based on the spectrum of HD 693, which has a high signal-to-noise above 300. We get $\log \varepsilon_{\odot}(\mathrm{S})=7.20$ (excluding the $\lambda 604.60 \mathrm{~nm}$ line), which is the same as the meteorite value (Grevesse \& Sauval 1998). We emphasize that the inclusion of this line or not in the abundance analysis for stars will not change the result of this work because it gives a consistent abundance with that derived from other lines. Experimental $\log g f$ values for five Si I lines are taken from Garz (1973), and the differential $\log g f$ values for the remaining $\mathrm{Si}$ I lines are taken from Chen et al. (2000). The enhancement factor, $E_{\gamma}$, is assumed to be 2.5 for Fe II and S I and 1.3 for $\mathrm{Si}$ I lines. There is no significant difference in the derived sulphur and iron abundances if $E_{\gamma}$ is changed from 2.5 to 1.5 , because the $\mathrm{S}_{\mathrm{I}}$ and $\mathrm{Fe}$ II lines are weak. Si I lines are somewhat stronger, but the maximum effect on $[\mathrm{Si} / \mathrm{Fe}]$ for the most metal-rich star HD 1461 is only 0.05 dex when $E_{\gamma}=2.5$ is adopted instead of 1.3 .
In sum, we obtained mean solar values: $[\mathrm{Fe} / \mathrm{H}]=0.02$, $[\mathrm{S} / \mathrm{Fe}]=-0.02$ and $[\mathrm{Si} / \mathrm{Fe}]=-0.01$ relative to the meteorite abundances given by Grevesse \& Sauval (1998). Stellar abundances, presented in Table 1, are relative to these values, so that the solar ratios $[\mathrm{X} / \mathrm{Fe}]$ in the following figures are exactly zero.

\subsection{Abundance uncertainties}

In order to investigate which lines, Fe I or Fe II, are the best indicator for the iron abundance in deriving the $[\mathrm{S} / \mathrm{Fe}]$ ratio, we show in Table 3 the sensitivity of the derived abundances to the stellar parameters for a representative star HD 28620. This star has $T_{\text {eff }}=6114 \mathrm{~K}, \log g=4.11,[\mathrm{Fe} / \mathrm{H}]=-0.38$, which is typical for the range of temperature, gravity, and metallicity of our sample of stars. In these calculations, the log $g f$ values for Fe I lines are the same as those of Chen et al. (2000), and the deviations are obtained by a change of $100 \mathrm{~K}$ in effective temperature, 0.1 dex in gravity, 0.1 dex in metallicity, and $0.3 \mathrm{~km} \mathrm{~s}^{-1}$ in microturbulence.

Clearly, sulphur abundances with respect to iron abundances derived from $\mathrm{Fe}$ II lines are more immune to variations 
Table 2. The atomic line data for S I, Si I and Fe II lines, and solar flux equivalent widths and abundances. Note that the mean solar sulphur abundance is derived excluding the $\lambda 604.60 \mathrm{~nm}$ line.

\begin{tabular}{|c|c|c|c|c|c|c|c|}
\hline Ele & $\begin{array}{r}\lambda \\
\mathrm{nm}\end{array}$ & $\begin{array}{r}\chi_{l} \\
\mathrm{eV}\end{array}$ & $\log g f$ & ref. & $E_{\gamma}$ & $\begin{array}{l}E W \\
\mathrm{~m} \AA\end{array}$ & Abu. \\
\hline $\mathrm{S}_{\mathrm{I}}$ & 604.603 & 7.87 & -0.51 & 1 & 2.5 & 14.5 & $7.32:$ \\
\hline$S_{\text {I }}$ & 605.267 & 7.87 & -0.63 & 2 & 2.5 & 9.8 & 7.25 \\
\hline $\mathrm{S}_{\mathrm{I}}$ & 675.717 & 7.87 & -0.31 & 2 & 2.5 & 16.1 & 7.21 \\
\hline$S_{\text {I }}$ & 869.396 & 7.87 & -0.52 & 2 & 2.5 & 11.0 & 7.19 \\
\hline$S_{\text {I }}$ & 869.464 & 7.87 & 0.05 & 2 & 2.5 & 28.9 & 7.17 \\
\hline \multicolumn{8}{|c|}{ Mean $\log \varepsilon_{\odot}(S)=7.20 \pm 0.03$} \\
\hline Si I & 566.556 & 4.92 & -2.04 & 3 & 1.3 & 40.9 & 7.57 \\
\hline Si I & 569.043 & 4.93 & -1.87 & 3 & 1.3 & 53.8 & 7.62 \\
\hline Si I & 570.111 & 4.93 & -2.05 & 3 & 1.3 & 37.1 & 7.52 \\
\hline Si I & 570.840 & 4.95 & -1.40 & 1 & 1.3 & 76.0 & 7.49 \\
\hline Si I & 577.215 & 5.08 & -1.66 & 1 & 1.3 & 54.8 & 7.55 \\
\hline Si I & 579.308 & 4.93 & -1.95 & 1 & 1.3 & 47.2 & 7.59 \\
\hline Si I & 579.786 & 4.95 & -2.05 & 3 & 1.3 & 43.1 & 7.64 \\
\hline Si I & 594.855 & 5.08 & -1.19 & 1 & 1.3 & 87.1 & 7.57 \\
\hline Si I & 612.503 & 5.61 & -1.54 & 1 & 1.3 & 33.3 & 7.54 \\
\hline Si I & 614.249 & 5.62 & -1.48 & 1 & 1.3 & 38.7 & 7.59 \\
\hline $\mathrm{Si}$ I & 614.502 & 5.62 & -1.43 & 1 & 1.3 & 39.6 & 7.55 \\
\hline Si I & 703.491 & 5.87 & -0.81 & 1 & 1.3 & 69.6 & 7.57 \\
\hline Si I & 722.621 & 5.61 & -1.30 & 1 & 1.3 & 47.6 & 7.53 \\
\hline Si I & 740.579 & 5.61 & -0.68 & 1 & 1.3 & 93.6 & 7.54 \\
\hline Si I & 741.596 & 5.61 & -0.71 & 3 & 1.3 & 91.5 & 7.54 \\
\hline Si I & 791.838 & 5.95 & -0.54 & 1 & 1.3 & 95.3 & 7.65 \\
\hline Si I & 793.235 & 5.96 & -0.35 & 1 & 1.3 & 113.6 & 7.64 \\
\hline Si I & 872.802 & 6.18 & -0.36 & 1 & 1.3 & 91.2 & 7.53 \\
\hline \multicolumn{8}{|c|}{ Mean $\log \varepsilon_{\odot}(\mathrm{Si})=7.57 \pm 0.03$} \\
\hline Fe II & 599.138 & 3.15 & -3.61 & 4 & 2.5 & 31.9 & 7.52 \\
\hline Fe II & 608.410 & 3.20 & -3.86 & 4 & 2.5 & 20.7 & 7.52 \\
\hline Fe II & 611.332 & 3.22 & -4.16 & 1 & 2.5 & 11.3 & 7.50 \\
\hline Fe II & 614.925 & 3.89 & -2.77 & 4 & 2.5 & 37.5 & 7.50 \\
\hline Fe II & 617.940 & 5.57 & -2.65 & 4 & 2.5 & 3.2 & 7.50 \\
\hline Fe II & 623.995 & 3.89 & -3.49 & 4 & 2.5 & 13.3 & 7.53 \\
\hline Fe II & 624.756 & 3.89 & -2.38 & 4 & 2.5 & 56.4 & 7.52 \\
\hline $\mathrm{Fe}$ II & 638.371 & 5.55 & -2.32 & 4 & 2.5 & 7.2 & 7.54 \\
\hline $\mathrm{Fe}$ II & 641.693 & 3.89 & -2.79 & 4 & 2.5 & 40.7 & 7.59 \\
\hline Fe II & 643.268 & 2.89 & -3.63 & 5 & 2.5 & 41.4 & 7.49 \\
\hline $\mathrm{Fe}$ II & 645.639 & 3.90 & -2.13 & 4 & 2.5 & 65.2 & 7.46 \\
\hline Fe II & 651.608 & 2.89 & -3.38 & 1 & 2.5 & 54.0 & 7.50 \\
\hline Fe II & 722.240 & 3.89 & -3.35 & 4 & 2.5 & 18.9 & 7.57 \\
\hline Fe II & 722.446 & 3.89 & -3.29 & 4 & 2.5 & 19.7 & 7.54 \\
\hline Fe II & 744.933 & 3.89 & -3.22 & 4 & 2.5 & 21.1 & 7.51 \\
\hline $\mathrm{Fe}$ II & 747.970 & 3.89 & -3.64 & 4 & 2.5 & 9.7 & 7.49 \\
\hline $\mathrm{Fe}$ II & 751.583 & 3.90 & -3.48 & 4 & 2.5 & 13.1 & 7.50 \\
\hline Fe II & 771.173 & 3.90 & -2.59 & 4 & 2.5 & 49.3 & 7.54 \\
\hline \multicolumn{8}{|c|}{ Mean $\log \varepsilon_{\odot}(\mathrm{Fe})=7.52 \pm 0.04$} \\
\hline
\end{tabular}

References for $\log g f$ values: (1) differential values in this work; (2) Kurucz 2002; (3) Garz 1973; (4) Biémont et al. 1991; (5) Hannaford et al. 1992.

of atmospheric parameters as compared to sulphur relative to iron from Fe I lines. This is primarily due to the high ionization potential of sulphur $(10.36 \mathrm{eV})$, which means that sulphur mainly occurs as S I atoms at temperatures of $\sim 6000 \mathrm{~K}$. Thus, we adopt the iron abundance derived from Fe II lines (hereafter the iron abundance) for all stars. Since the total error of [Si/Fe]
Table 3. Dependences of relative abundances on the stellar parameters.

\begin{tabular}{|c|c|c|c|c|}
\hline \multicolumn{2}{|c|}{$T_{\text {eff }}=6114 \mathrm{~K}, \log g=4.11,[\mathrm{Fe} / \mathrm{H}]=-0.38, \xi_{\mathrm{t}}=1.6 \mathrm{~km} \mathrm{~s}^{-1}$} & & & \\
\hline & $T_{\text {eff }}$ & $\log g$ & {$[\mathrm{Fe} / \mathrm{H}]$} & $\xi_{\mathrm{t}}$ \\
\hline & $+100 \mathrm{~K}$ & $+0.1 \mathrm{dex}$ & $+0.1 \mathrm{dex}$ & $+0.3 \mathrm{~km} \mathrm{~s}^{-1}$ \\
\hline$[\mathrm{Fe} \mathrm{I} / \mathrm{H}]$ & 0.07 & 0.00 & 0.00 & -0.05 \\
\hline$[\mathrm{Fe} \mathrm{II} / \mathrm{H}]$ & -0.01 & 0.04 & 0.01 & -0.04 \\
\hline$[\mathrm{S} \mathrm{I} / \mathrm{Fe} \mathrm{I}]$ & -0.12 & 0.03 & 0.00 & 0.05 \\
\hline$[\mathrm{S}$ I/Fe II $]$ & -0.04 & -0.01 & -0.01 & 0.04 \\
\hline$[\mathrm{Si} / \mathrm{Fe} \mathrm{I}]$ & -0.04 & 0.00 & 0.00 & -0.03 \\
\hline$[\mathrm{Si} / \mathrm{Fe} \mathrm{II}]$ & 0.04 & -0.04 & -0.01 & -0.02 \\
\hline
\end{tabular}

ratio is only a bit larger when $\mathrm{Si}$ is measured relative to $\mathrm{Fe}$ derived from Fe II lines instead of Fe I lines (see Table 3), both silicon and sulphur abundances are relative to iron abundances from Fe II lines. A further advantage of using Fe II lines is that non-LTE effects on $[\mathrm{Fe} / \mathrm{H}]$ are bound to be small because nearly all iron occur as Fe II ions - a conclusion which is supported by detailed calculations by Thévenin \& Idiart (1999).

The line-to-line scatter of the deduced abundances gives an estimate of the uncertainty arising from the measured equivalent widths. For silicon and iron, more than 10 lines are available for most stars and the statistical error of the mean abundance is less than 0.02 dex. With 2-5 sulphur lines used in this work, the corresponding error of the mean abundance is of the order of 0.03 dex. Taking into account the errors from the uncertainties of the stellar parameters in Table 3, we estimate the total errors to be $\sim 0.06$ dex for $[\mathrm{Fe} / \mathrm{H}]$ and $[\mathrm{Si} / \mathrm{Fe}]$, and $\sim 0.07$ dex for $[\mathrm{S} / \mathrm{Fe}]$; the corresponding error bars are shown in Fig. 3.

The above error estimates do not, however, account for possible systematic errors introduced for example by the assumption of LTE or the use of 1D hydrostatic model atmospheres. The non-LTE effect on the S I $\lambda 869.39 \mathrm{~nm}$ and $\lambda 869.46 \mathrm{~nm}$ lines has been investigated by Takada-Hidai et al. (2002), who showed that this effect is negligible. For the other three $\mathrm{S}$ I lines, non-LTE effects are also expected to be insignificant because they have the same excitation potential as the $\lambda 869.46 \mathrm{~nm}$ line and they are weaker. As mentioned above, iron abundances derived from $\mathrm{Fe}$ II lines are generally immune to non-LTE effects. Thus, non-LTE effects are expected to be negligible in the trend of $[\mathrm{S} / \mathrm{Fe}]$ versus $[\mathrm{Fe} / \mathrm{H}]$.

Following the same procedure as in Asplund et al. (1999) and Asplund \& García Pérez (2001), we have estimated the effects of stellar granulation on the derived $\mathrm{S}$ and $\mathrm{Si}$ abundances using the new generation of ab-initio 3D hydrodynamical model atmospheres (e.g. Asplund et al. 2000). A strictly differential comparison of the predicted line strengths in the 3D models and 1D MARCS model atmospheres with identical parameters has been performed for $\mathrm{S}_{\mathrm{I}}$ and $\mathrm{Si}$ I lines; the results for Fe II lines have been culled from Asplund \& García Pérez (2001). As seen in Table 4, the 1D results are relatively little affected due to the high excitation potential of the $S_{\text {I }}$ and Si I lines which forces them to be formed at similar atmospheric 
Table 4. Comparison of the $\mathrm{Si}, \mathrm{S}$ and Fe LTE abundances derived with $1 \mathrm{D}$ hydrostatic and 3D hydrodynamical model atmospheres. The 3D abundances are those which reproduce the equivalent widths computed using a 1D model atmosphere with identical parameters, a microturbulence of $\xi_{\text {turb }}=1.0 \mathrm{~km} \mathrm{~s}^{-1}$ and the abundances given in the third and fourth columns

\begin{tabular}{lccccc}
\hline \hline $\begin{array}{l}T_{\text {eff }} \\
{[\mathrm{K}]}\end{array}$ & $\begin{array}{c}\log g \\
{[\mathrm{cgs}]}\end{array}$ & {$[\mathrm{Fe} / \mathrm{H}]$} & {$[\alpha / \mathrm{Fe}]^{\mathrm{a}}$} & $\Delta(\mathrm{S} / \mathrm{Fe})^{\mathrm{b}}$ & $\Delta(\mathrm{Si} / \mathrm{Fe})^{\mathrm{c}}$ \\
\hline 5767 & 4.44 & +0.0 & +0.00 & -0.02 & -0.01 \\
5822 & 4.44 & -1.0 & +0.40 & -0.03 & -0.04 \\
6191 & 4.04 & +0.0 & +0.00 & +0.02 & -0.01 \\
6180 & 4.04 & -1.0 & +0.40 & +0.01 & -0.03 \\
\hline
\end{tabular}

a The $\alpha$-enhancement for the S and Si abundances. For these calculations, $\log \epsilon(\mathrm{S})=7.33, \log \epsilon(\mathrm{Si})=7.55$ and $\log \epsilon(\mathrm{Fe})=7.50$ have been assumed for the Sun following Grevesse \& Sauval (1998).

b Based on the five $\mathrm{S}_{\mathrm{I}}$ lines listed in Table 2 and the six Fe II lines used by Asplund \& García Pérez (2001).

c Based on the four Si I 570.1, 594.8, 722.6 and $872.8 \mathrm{~nm}$ lines and the six Fe II lines used by Asplund \& García Pérez (2001).

depths as the Fe II lines. For the relevant metallicity range, the $\mathrm{S} / \mathrm{Fe}$ and $\mathrm{Si} / \mathrm{Fe}$ ratios differ by $\leq 0.04$ dex when using $3 \mathrm{D}$ model atmospheres compared to the $1 \mathrm{D}$ case. To avoid interpolation within the still limited sample of granulation corrections and given their minor impact on the final results, we have made no attempt to apply such corrections to the 1D abundances of the individual stars in the following discussion.

\section{Results and discussions}

\subsection{Sulphur abundances vs. metallicity}

The $[\mathrm{S} / \mathrm{Fe}]$ ratio as a function of metallicity is shown in Fig 3. It seems that the $[\mathrm{S} / \mathrm{Fe}]$ ratio is nearly solar for $[\mathrm{Fe} / \mathrm{H}]>-0.3$, below which it increases with decreasing metallicity and an overabundance of $\sim 0.25 \mathrm{dex}$ is indicated at $[\mathrm{Fe} / \mathrm{H}]=-1.0$. There may be a tendency for decreasing $[\mathrm{S} / \mathrm{Fe}]$ for super metal-rich stars but this is based on one star with $[\mathrm{Fe} / \mathrm{H}] \sim+0.5$ only. These results are in agreement with those of François (1987, 1988 ), but the scatter in the $[\mathrm{S} / \mathrm{Fe}]$ ratio at a given metallicity is significantly smaller in the present work.

\subsection{Reanalysis of data from François $(1987,1988)$}

Israelian \& Rebolo (2001) made a reanalysis of the data presented in François $(1987,1988)$ by using a new determination of stellar parameters and suggest a linear increase of the $[\mathrm{S} / \mathrm{Fe}]$ ratio with decreasing metallicity. One point in their paper is, however, strange: stars with $[\mathrm{Fe} / \mathrm{H}]>-0.4$ have $[\mathrm{S} / \mathrm{Fe}]$ around -0.3 , which is significantly lower than the solar ratio. At the same time, they derived a solar sulphur abundance of 7.20, which is the same as the meteoritic value. Furthermore, the scatter in $[\mathrm{S} / \mathrm{Fe}]$ obtained by Israelian \& Rebolo (2001) is significantly larger than in the present work.

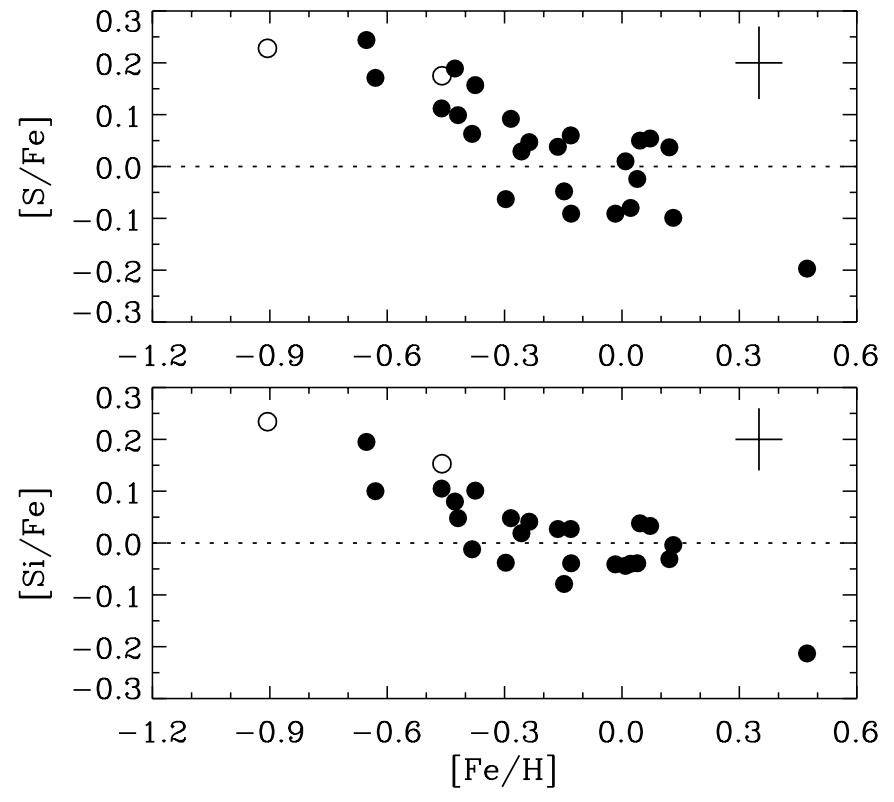

Fig. 3. The $[\mathrm{S} / \mathrm{Fe}]$ and $[\mathrm{Si} / \mathrm{Fe}]$ ratio as a function of metallicity. Filled circles denote the thin disk stars while open circles represent stars with kinematics typical for the thick disk.

Table 5. Stellar parameters and relative abundances for the reanalysis of data from François $(1987,1988)$.

\begin{tabular}{rcccccc}
\hline \hline $\mathrm{HD}$ & $\begin{array}{c}T_{\text {eff }} \\
\mathrm{K}\end{array}$ & $\log g$ & $\begin{array}{c}\xi_{\mathrm{t}} \\
\mathrm{km} \mathrm{s}^{-1}\end{array}$ & $\begin{array}{c}{[\mathrm{Fe} / \mathrm{H}]} \\
\text { LTE }\end{array}$ & $\begin{array}{c}{[\mathrm{Fe} / \mathrm{H}]} \\
\text { NLTE }\end{array}$ & $\begin{array}{c}{[\mathrm{S} / \mathrm{H}]} \\
\text { LTE }\end{array}$ \\
\hline 59984 & 5896 & 3.93 & 1.6 & -0.88 & -0.74 & -0.67 \\
63077 & 5825 & 4.15 & 1.2 & -0.89 & -0.75 & -0.57 \\
69897 & 6227 & 4.20 & 1.6 & -0.50 & -0.41 & -0.41 \\
76932 & 5870 & 4.08 & 1.3 & -0.98 & -0.83 & -0.63 \\
88218 & 5661 & 3.94 & 1.4 & -0.53 & -0.44 & -0.26 \\
91324 & 6123 & 3.95 & 1.8 & -0.60 & -0.50 & -0.49 \\
102365 & 5562 & 4.39 & 1.0 & -0.39 & -0.32 & -0.35 \\
106516 & 6135 & 4.30 & 1.5 & -0.79 & -0.66 & -0.55 \\
136352 & 5584 & 4.27 & 1.0 & -0.58 & -0.48 & -0.44 \\
139211 & 6231 & 4.12 & 1.7 & -0.26 & -0.21 & -0.16 \\
148816 & 5784 & 4.07 & 1.4 & -1.03 & -0.87 & -0.54 \\
157089 & 5712 & 4.00 & 1.4 & -0.79 & -0.66 & -0.44 \\
193901 & 5662 & 4.48 & 1.0 & -1.13 & -0.96 & -0.67 \\
201891 & 5825 & 4.21 & 1.3 & -1.21 & -1.03 & -0.86 \\
203608 & 6094 & 4.29 & 1.4 & -0.91 & -0.77 & -0.69 \\
\hline
\end{tabular}

In order to investigate possible systematic differences relative to the work by Israelian \& Rebolo (2001), we have made a reanalysis of sulphur and iron abundances for 15 François $(1987,1988)$ stars with similar stellar parameters as our sample stars, i.e. $5500<T_{\text {eff }}<6600 \mathrm{~K}, \log g>3.8$ and $[\mathrm{Fe} / \mathrm{H}]>-1.1$. The reanalysis was performed in the same way as that for our sample of stars, with parameters derived from Strömgren photometry and Hipparcos parallaxes. The microturbulence was estimated from the relation presented in Edvardsson et al. (1993) and the equivalent widths of Fe I 


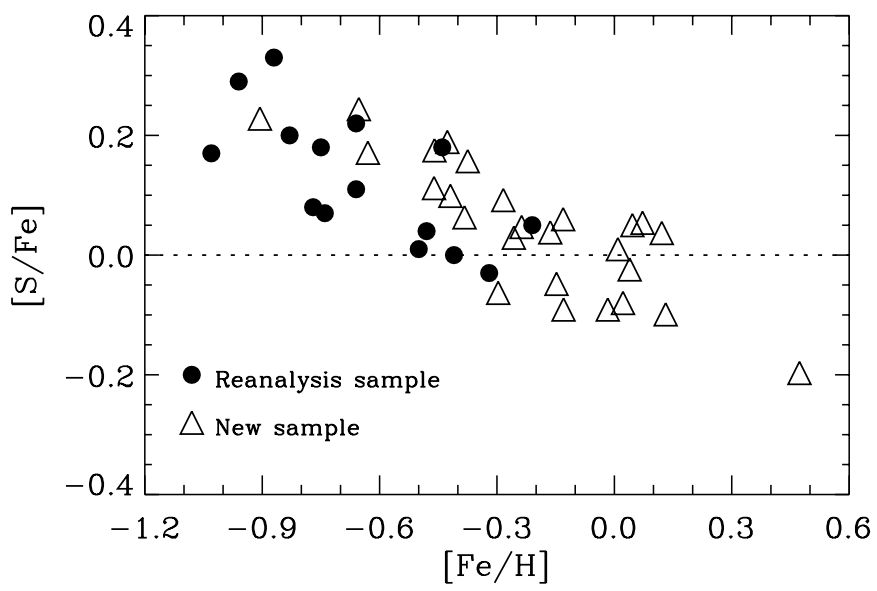

Fig. 4. The $[\mathrm{S} / \mathrm{Fe}]$ vs. $[\mathrm{Fe} / \mathrm{H}]$ trend for the new measurements (open triangles) and our reanalysis of the François $(1987,1988)$ data (filled circles).

lines published by François $(1987,1988)$ were used to derive iron abundances. Adopted $\log g f$ values are -1.68 for Fe I $867.48 \mathrm{~nm}$ and -0.38 for Fe I $869.95 \mathrm{~nm}$ taken from Nave et al. (1994); the very strong Fe I $868.86 \mathrm{~nm}$ line was discarded because of its sensitivity to the uncertain damping constant. The solar equivalent widths for the Fe I $867.48 \mathrm{~nm}$ and Fe I $869.95 \mathrm{~nm}$ lines were measured from the solar flux spectrum by Kurucz et al. (1984). Again, stellar abundances (see Table 5) are relative to the Sun so that uncertainties from atomic line data cancel out. The LTE iron abundances derived from Fe I lines were corrected for non-LTE effects using the calculations of Thévenin \& Idiart (1999). These non-LTE iron abundances are more consistent with those derived from Fe II lines for our new sample of stars. As shown in Fig. 4, the scatter in the $[\mathrm{S} / \mathrm{Fe}]$ vs. $[\mathrm{Fe} / \mathrm{H}]$ trend at a given metallicity is quite small and the results from the reanalysis of the François data and the new measurements in the present work are consistent.

Figure 5 shows the deviations of $[\mathrm{S} / \mathrm{H}]$ and $[\mathrm{Fe} / \mathrm{H}]$ between the present work and Israelian \& Rebolo (2001) for 14 stars in common. One of the 15 stars in our reanalysis sample was not included in Israelian \& Rebolo (2001). It is clear that there is no significant deviation for the sulphur abundances between the two works, but the iron abundances show large differences. The iron abundances adopted by Israelian \& Rebolo (2001) for 10 François $(1987,1988)$ stars are significantly higher than the iron abundances in the present work, which explains the low $[\mathrm{S} / \mathrm{Fe}]$ ratio $(-0.3)$ they find at solar metallicity. We note that they adopt $[\mathrm{Fe} / \mathrm{H}]$ values from different sources in the literature. Therefore, the iron abundances may be inconsistent with $T_{\text {eff }}$ and $\log g$, and consequently also with the derived $[\mathrm{S} / \mathrm{H}]$. This explains both the large scatter and the offset with respect to the Sun in Fig. 3 of Israelian \& Rebolo (2001), emphasizing the need for a proper and consistent treatment of the different species used to derive abundance ratios such as $[\mathrm{S} / \mathrm{Fe}]$. The adopted stellar parameters are, otherwise, quite similar for the two works.

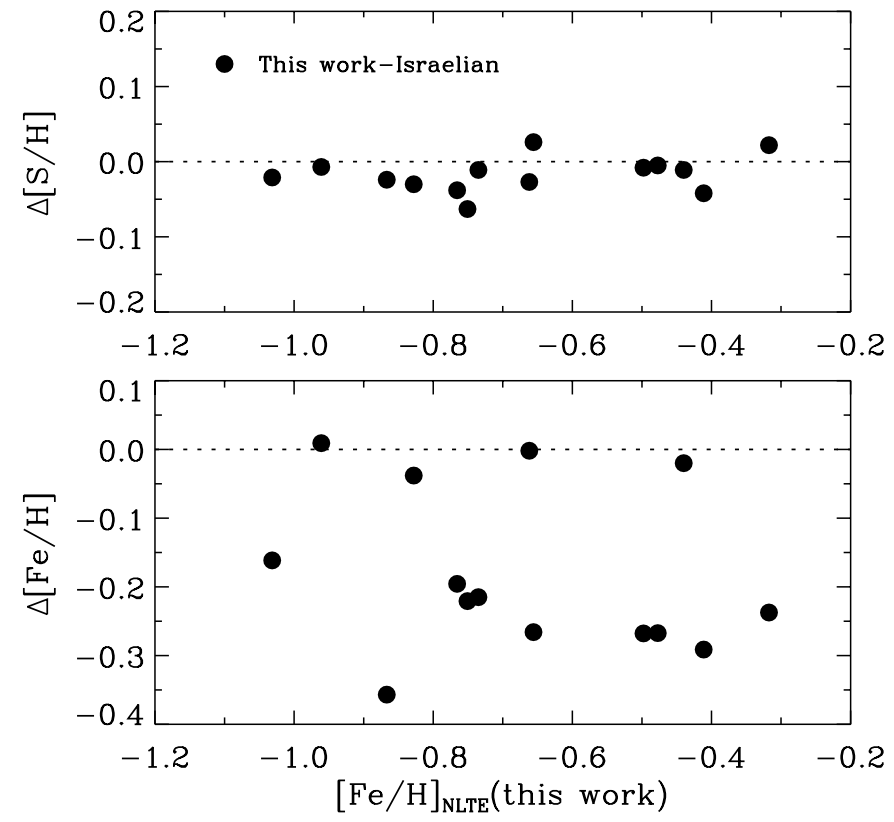

Fig. 5. Abundance comparison between this work and Israelian \& Rebolo (2001) for $[\mathrm{S} / \mathrm{H}]$ and $[\mathrm{Fe} / \mathrm{H}]$ based on the reanalysis of the François $(1987,1988)$ stars.

\subsection{Correlation between sulphur and silicon abundances}

As described in the Introduction, one goal of this work is the comparison of sulphur with other $\alpha$-capture elements in particular the closest element, silicon, being a good representative. The second reason for the choice of silicon is that we have many good silicon lines in the spectra and the oscillator strengths are quite reliable; abundances derived from different lines are consistent for both the Sun and stars. Thus we can expect that $[\mathrm{Si} / \mathrm{Fe}]$ vs. $[\mathrm{Fe} / \mathrm{H}]$ will be well defined.

As shown in Fig. 3, the trend of $[\mathrm{Si} / \mathrm{Fe}]$ vs. $[\mathrm{Fe} / \mathrm{H}]$ is similar to that of sulphur and the scatter at a given metallicity is also the same. The one-to-one relation of $[\mathrm{S} / \mathrm{H}]$ vs. $[\mathrm{Si} / \mathrm{H}]$ is shown in Fig. 6; there is a very good correlation between sulphur and silicon indicating common nucleosynthesis sites for these two elements.

In order to investigate the dependence of the correlation of sulphur and silicon on metallicity, we plot the $[\mathrm{S} / \mathrm{Si}]$ ratio as a function of $[\mathrm{Fe} / \mathrm{H}]$ in the bottom of Fig. 6. It seems that the sulphur abundance relative to silicon is constant for the whole $[\mathrm{Fe} / \mathrm{H}]$ range investigated in this work.

Goswami \& Prantzos (2000) investigated the chemical evolution of many elements based on the yields of Woosley \& Weaver (1995) for two cases: Case A considered the constant (solar) metallicity yields while Case B corresponds to metallicity dependent yields. They found exactly the same trend for the sulphur and silicon evolution in Case A and Case B in the whole metallicity range of $-4.0<[\mathrm{Fe} / \mathrm{H}]<+0.5$. They suggested that the elements $\mathrm{O}, \mathrm{Si}, \mathrm{S}$ and $\mathrm{Ca}$, behave as true primaries, whithout any metallicity dependence of their yields. The predicted ratios of S and Si in Goswami \& Prantzos (2000) 

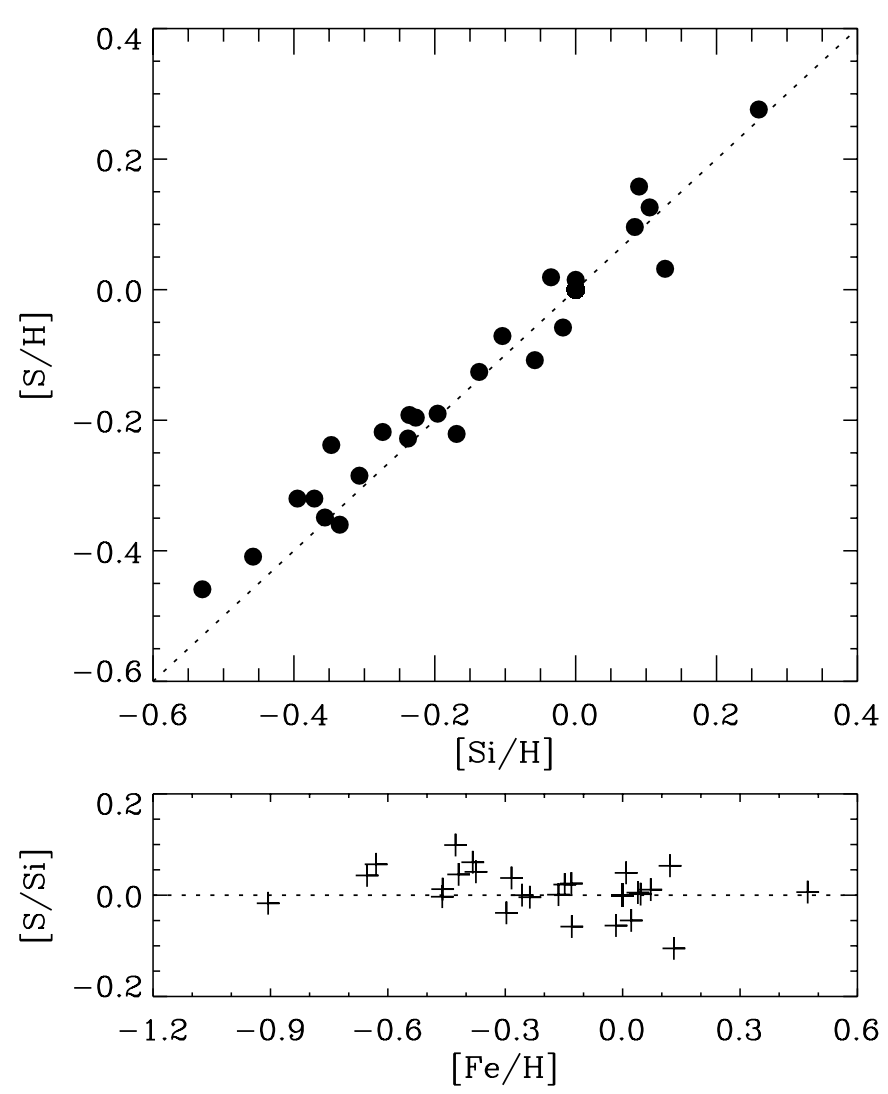

Fig. 6. The correlation between $[\mathrm{S} / \mathrm{H}]$ and $[\mathrm{Si} / \mathrm{H}]$, and the $[\mathrm{S} / \mathrm{Si}]$ ratio as a function of metallicity.

and other chemical evolution models, e.g. Timmes et al. (1995), Chiappini et al. (1997) and Samland et al. (1998), are consistent with the results of the present work for disk stars.

\subsection{Kinematics}

Stellar kinematics are useful information to interpret the stellar abundances and to probe the origin of the stars. We calculated the kinematics for our program stars based on proper motions and parallaxes in the Hipparcos survey and the radial velocity derived from the Doppler shift of the spectral lines in this work. The calculation of galactic space velocity $(U, V, W)$ follows that presented in Johnson \& Soderblom (1987). The correction of space velocity to the Local Standard of Rest is based on a solar motion, $(-10.0,+5.2,+7.2) \mathrm{km} \mathrm{s}^{-1}$ in $(U, V, W)$ as derived from Hipparcos data by Dehnen \& Binney (1998).

As shown in Table 1, most of the sample stars are thin disk stars $\left(V_{\mathrm{LSR}}>-40 \mathrm{~km} \mathrm{~s}^{-1}\right)$. Exceptions are HD 10453 and HD 22879, which have thick disk kinematics. The [S/Fe] ratio is about 0.2 dex for both stars, which is higher than most other stars. These data seem to be in line with the result by Edvardsson et al. (1993), who found an abundance gradient of $\alpha$ elements at the metallicity of $[\mathrm{Fe} / \mathrm{H}] \sim-0.7$. But the metallicities of the two thick disk stars are also the lowest, and thin disk kinematics does not imply a low $[\mathrm{S} / \mathrm{Fe}]$ ratio. For example, HD 77967 and HD 159307 have thin disk kinematics, but both $[\mathrm{S} / \mathrm{Fe}]$ and $[\mathrm{Si} / \mathrm{Fe}]$ are close to the enhanced value of $\sim 0.2$ dex.
Therefore, it seems that these abundances ratios are primarily a function of metallicity and the kinematics plays a minor role.

\section{Conclusions}

We have carried out new measurements of sulphur and silicon abundances for 26 main sequence stars in the metallicity range of $-1.0<[\mathrm{Fe} / \mathrm{H}]<+0.5$ and have established the trends of $[\mathrm{Si} / \mathrm{Fe}]$ and $[\mathrm{S} / \mathrm{Fe}]$ with a small scatter at a given metallicity. The result indicates a very good correlation between sulphur and silicon and the correlation does not depend on the metallicity. The results provide support for the chemical evolution models of Goswami \& Prantzos (2000), Timmes et al. (1995), Chiappini et al. (1997) and Samland et al. (1998) for these two elements in the case of disk stars. It is concluded that the nucleosynthesis of sulphur is a proxy for silicon, which is the nearest $\alpha$-capture element of sulphur in the periodic table of elements.

Acknowledgements. This research was supported mainly by the Danish Rectors' Conference and the Chinese Academy of Sciences and partly by the NKBRSF G1999075406 and the NSFC under grant 10173014.

\section{References}

Alonso, A., Arribas, S., \& Martínez-Roger, C. 1995, A\&A, 297, 197 Alonso, A., Arribas, S., \& Martínez-Roger, C. 1996, A\&A, 313, 873 Asplund, M., Gustafsson, B., Kiselman, D., \& Eriksson, K. 1997, A\&A, 318, 521

Asplund, M., Nordlund, Å., Trampedach, R., \& Stein, R. F. 1999, A\&A, 346, L17

Asplund, M., Nordlund, ̊̊., Trampedach, R., et al. 2000, A\&A, 359, 729

Asplund, M., \& García Pérez, A. E. 2001, A\&A, 372, 601

Biémont, E., Baudoux, M., Kurucz, R. L., et al. 1991, A\&A, 249, 539

Boesgaard, A. M., King, J. R., Deliyannis, C. P., \& Vogt, S. S. 1999, AJ, 117, 492

Centurión, M., Bonifacio, P., Molaro, P., \& Vladilo, G. 2000, ApJ, 536, 540

Chen, Y. Q., Nissen, P. E., Zhao, G., et al. 2000, A\&AS, 141, 491

Chiappini, C., Matteucci, F., \& Gratton, R. 1997, ApJ, 477, 765

Clegg, R. E. S., Lambert, D. L., \& Tomkin, J. 1981, ApJ, 250, 262

Dehnen, W., \& Binney, J. J. 1998, MNRAS, 298, 387

ESA 1997, The Hipparcos and Tycho Catalogues, ESA SP-1200

Edvardsson, B., Andersen, J., Gustafsson, B., et al. 1993, A\&A, 275, 101

Finlator, K., Ivezic, Z., Fan, X., et al. 2000, AJ, 120, 2615

François, P. 1987, A\&A, 176, 294

François, P. 1988, A\&A, 195, 226

Fuhrmann, K. 1998, A\&A, 338, 161

Garz, T. 1973, A\&A, 26, 471

Goswami, A., \& Prantzos, N. 2000, A\&A, 359, 191

Grevesse, N., \& Sauval, A. J. 1998, Space Sci. Rev., 85, 161

Hannaford, P., Lowe, R. M., Grevesse, N., \& Noels, A. 1992, A\&A, 259, 301

Idiart, T. P., \& Thévenin, F. 2000, ApJ, 541, 207

Israelian, G., Garcia Lopez, R., \& Rebolo, R. 1998, ApJ, 507, 805

Israelian, G., Rebolo, R., Garcia Lopez, R., et al. 2001, ApJ, 551, 833

Israelian, G., \& Rebolo, R. 2001, ApJ, 557, L43

Johnson, D. R. H., \& Soderblom, D. R. 1987, AJ, 93, 864

Kurucz, R. L. 2002, Atomic line list, available at his homepage (http://cfaku5.harvard.edu/linelists) 
Kurucz, R. L., Furenlid, I., Brault, J., \& Testerman, L. 1984, Solar Flux Atlas from 296 to $1300 \mathrm{~nm}$, National Solar Observatory, Sunspot, New Mexico

Nave, G., Johansson, S., Learner, R. C. M., et al. 1994, ApJS, 94, 221

Nissen, P. E., \& Edvardsson, B. 1992, A\&A, 261, 255

Nissen, P. E., Primas, F., \& Asplund, M. 2001, New Astron. Rev., 45, 545

Nissen, P. E., Asplund, M., Chen, Y. Q., \& Pettini, M. 2002, in preparation

Olsen, E. H. 1983, A\&AS, 54, 55

Olsen, E. H. 1988, A\&A, 189, 173

Olsen, E. H. 1993, A\&A, 102, 89
Ryan, S. G., Norris, J. E., \& Beers, T. C. 1996, ApJ, 471, 254

Samland, M. 1998, ApJ, 496, 155

Schuster, W. J., \& Nissen, P. E. 1989, A\&A, 221, 65

Stephens, A., \& Boesgaard, A. M. 2002, AJ, 123, 1647

Takada-Hidai, M., Takeda, Y., Sato, S., et al. ApJ, in press [astro-ph/0103481]

Thévenin, F., \& Idiart, T. P. 1999, ApJ, 521, 753

Timmes, F. X., Woosley, S. E., \& Weaver, T. 1995, ApJS, 98, 617

VandenBerg, D. A., Swenson, F. J., Rogers, F. J., Iglesias, C. A., \& Alexander, D. R. 2000, ApJ, 532, 430

Woosley, S. E., \& Weaver, T. A. 1995, ApJS, 101, 181 